\title{
Tratamento médico da tromboflebite superficial do membro inferior: heparina ou anti-inflamatórios?
}

Philippe Botas, ${ }^{*}$ José Pimenta, ${ }^{* *}$ Pedro Gonçalo Santos, ${ }^{* * *}$ Luiz Miguel Santiago,****

Objectivos: Clarificar qual a melhor terapêutica da tromboflebite superficial (TS) do membro inferior, com principal enfoque na comparação entre anti-inflamatórios (AINE) e heparina de baixo peso molecular (HBPM).

Fontes de dados: Base de dados Pubmed, sites de medicina baseada na evidência, Direcção-Geral de Saúde, Associação Portuguesa de Medicina Geral e Familiar, MGFamiliar.net e Índex de Revistas Médicas Portuguesas.

Métodos de revisão: Pesquisa de normas de orientação clínica (NOC), sistemas computorizados de apoio à decisão (SCAD), revisões sistemáticas (RS) e artigos originais, publicados entre Janeiro/2008 e Maio/2011, utilizando os termos MeSH: venous thrombosis; heparin, low-molecular-weight; anti-inflammatory agents. Para avaliar o nível de evidência, foi utilizada a escala Strength of Recommendation Taxonomy (SORT) da American Family Physician.

Resultados: Foram obtidos 215 artigos, seleccionando-se uma NOC, um SCAD, uma RS e um ensaio clínico aelatorizado (ECA). As evidências sugerem que existe melhoria dos sintomas dos doentes com TS tratados com HBPM ou AINE em comparação com placebo, reduzindo a incidência de recorrências e complicações, sem diferenças no perfil de segurança a curto prazo (nível de evidência 2). Também destacam a anticoagulação como terapêutica de primeira linha (nível de evidência 2). A utilização simultânea de HBPM e AINE revelou maior eficácia no alívio sintomático do que a HBPM isolada num ECA (nível de evidência 2).

Conclusões: A HBPM e os AINE são duas opções terapêuticas com evidências que suportam a sua utilização como primeira linha de tratamento (SOR B). A anticoagulação durante pelo menos 4 semanas está indicada sobretudo quando se identificam critérios de gravidade: proximidade anatómica do trombo com o sistema venoso profundo e factores de risco médicos para trombose venosa profunda (SOR B). São necessários mais ECA, sobretudo no que se relaciona com a opção por AINE ou HBPM ou a sua utilização simultânea, doses e duração de tratamento.

Palavras-chave: Tromboflebite; Heparina; Anti-inflamatórios; Terapêutica.

\section{INTRODUÇÃO}

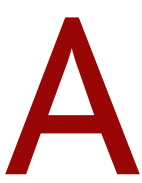
tromboflebite superficial (TS) é uma condição clínica frequente, que pode ser motivo de consulta na prática diária. Estima-se que tenha uma incidência entre 3 a $11 \%$, com maior frequência no sexo feminino e afectando predominantemente os membros inferiores (MI). ${ }^{1}$ É uma entidade clínica pouco estudada, com escassas referências em

*MD, Interno de Medicina Geral e Familiar, Centro de Saúde de Eiras **MD, Interno de Medicina Geral e Familiar, Centro de Saúde de Penacova ***MD, Interno de Medicina Geral e Familiar, Centro de Saúde Norton de Matos ****MD PhD, Assistente Graduado Sénior de Medicina Geral e Familiar, Centro de Saúde de Eiras
Portugal, existindo controvérsia sobre a sua abordagem terapêutica. O termo tromboflebite é largamente utilizado na literatura e frequentemente refere-se à inflamação venosa, mesmo quando não é certa a presença de trombose. ${ }^{2}$ Os autores optaram por utilizar este termo e conceito ao longo do trabalho de revisão.

Considerada durante muito tempo uma entidade de limitada relevância clínica, estudos recentes reconhecem a importância da TS em relação com as suas possíveis complicações [probabilidade de evolução para trombose venosa profunda (TVP), com o subsequente risco acrescido de tromboembolia pulmonar (TEP)], e como pista diagnóstica de outras patologias. ${ }^{2}$ Num es- 
tudo em que foram incluídos 844 doentes com TS sintomática dos MI com pelo menos $5 \mathrm{~cm}$ de extensão avaliada ecograficamente, 210 (24,9\%) também tinham TVP ou TEP sintomática. Nos restantes casos, 58 (10,2\%) desenvolveram complicações tromboembólicas (3 com TEP, 15 com TVP, 18 com progressão de TS e 10 com recorrência de TS). ${ }^{3}$

O risco de TVP é mais elevado quando a TS afecta o território proximal da veia safena interna, diagnosticando-se por ecografia em 6 a $53 \%$ dos casos. ${ }^{4} \mathrm{~A}$ evidência científica estabelece uma associação entre TS e tromboembolismo venoso, destacando-se factores de risco semelhantes: veias varicosas, imobilização prolongada, pós-operatório, traumatismo, gravidez e puerpério, neoplasias malignas, trombofilias, doenças autoimunes, história prévia de tromboembolismo venoso, contraceptivos orais, terapêutica de substituição hormonal e obesidade. ${ }^{5} \mathrm{~A}$ incidência de TVP é menor nas TS associadas a varizes comparativamente às associadas a outras patologias (3-20\% versus $44-60 \%$, respectivamente). ${ }^{6}$ Os factores de risco para extensão da TS ao sistema venoso profundo são a proximidade anatómica do trombo com este sistema e factores de risco médicos para TVP. ${ }^{4}$

O quadro clínico da TS é facilmente suspeitável: dor localizada e, ao exame objectivo, apresentação de rubor, calor, um cordão venoso doloroso palpável, por vezes edema e eritema circundantes. ${ }^{7} \mathrm{O}$ tratamento desta patologia tem como objectivos principais o alívio da sintomatologia dolorosa local, a prevenção do desenvolvimento de TVP e TEP e a diminuição das recorrências. ${ }^{5}$

A controvérsia na escolha do tratamento da TS levou à elaboração desta revisão, com o objectivo de determinar qual a melhor opção terapêutica: anti-inflamatórios não esteróides (AINE) ou heparina de baixo peso molecular (HBPM).

\section{MÉTODOS}

Procedeu-se à pesquisa nas bases de dados National Guideline Clearinghouse, Guidelines Finder, Canadian Medical Association Practice Guidelines InfoBase, The Cochrane Library, Clinical Evidence, DARE, UpToDate e Pubmed, e ainda nos sites da Direcção-Geral de Saúde, Associação Portuguesa de Médicos de Medicina Geral e Familiar, MGFamiliar.net e no Índex de Revistas Médicas Portuguesas, utilizando os termos MeSH: venous thrombosis; heparin, low-molecular-weight e anti-inflammatory agents; e os descritores portugueses: «trombose venosa» $\mathrm{e}$ «tromboflebite». Limitou-se a pesquisa a normas de orientação clínica (NOC), sistemas computorizados de apoio à decisão (SCAD), revisões sistemáticas (RS) e ensaios clínicos aelatorizados (ECA), publicados entre 01/01/2008 a 20/05/2011.

Os critérios utilizados para a inclusão dos artigos nesta revisão foram os seguintes:

- População: indivíduos observados em ambulatório com o diagnóstico de TS no MI.

- Intervenção: Terapêutica com HBPM e/ou AINE.

- Outcomes: Alívio sintomático e prevenção de complicações.

Como critérios de exclusão foram definidos os seguintes: artigos repetidos; TVP isolada; TS em contexto de internamento; TS em zonas que não o MI; TS em idade pediátrica; TS na grávida; TS complicada ou associada a condições patológicas que aumentam o risco, nomeadamente neoplasias, coagulopatias e período pós-cirúrgico; profilaxia de TS.

Os autores adoptaram a escala SORT para classificação do nível de evidência/força de recomendação. ${ }^{8}$

\section{RESULTADOS}

Na pesquisa efectuada foram encontrados 215 artigos, dos quais 4 cumpriram os critérios de inclusão: uma NOC, um SCAD, uma RS e um ECA.

A NOC publicada pelo American College ofChest Physicians $^{9}$ em 2008 destaca, com uma força de recomendação B (após conversão para a SORT), que o tratamento da TS deve ser realizado com doses profilácticas ou intermédias de HBPM ou doses intermédias de heparina não fraccionada (HNF) por um período mínimo de 4 semanas. " Como alternativa, com uma menor força de recomendação (SOR C), é sugerida a terapêutica durante 4 semanas com antagonistas da vitamina $\mathrm{K}$ (AVK) (INR alvo 2-3), associando HBPM ou HNF nos primeiros 5 dias. É desaconselhada a associação de AINE à terapêutica anticoagulante, com força de recomendação B. Sem atribuição de um grau de recomendação, a NOC sugere que, em caso de TS menos grave (quando o segmento venoso afectado é curto em extensão ou

\footnotetext{
* Dose intermédia $=2 \times$ dose profilática. Por exemplo, dalteparina 5000 $\mathrm{UI}, \mathrm{SC}$, cada 12 horas ou enoxaparina $40 \mathrm{mg}$ (4000 UI), SC, cada 12 horas.
} 


QUADRO I. Sistema computorizado de apoio à decisão.
\begin{tabular}{|l|l|}
\hline Autores & Conclusões \\
\hline Fernandez L, et al. ${ }^{4}$ & $\begin{array}{l}\text { Recomendações } \\
- \text { Baixo risco TVP } \\
\text { UPToDate } 2011\end{array}$ \\
& $\begin{array}{l}\text { AINE (SOR B) } \\
\text { Anticoagulação por 4 semanas } \\
\text { (SOR B) }\end{array}$ \\
& $\begin{array}{l}\text { - Extensão ao SVP } \\
\text { Anticoagulação segundo protocolo } \\
\text { de tratamento de TVP }\end{array}$ \\
\hline
\end{tabular}

TVP - Trombose venosa profunda AINE - Anti-inflamatório não esteróide SVP - Sistema venoso profundo

distante da junção safenofemoral), não será necessário o tratamento anticoagulante, indicando o uso de AINE por via oral ou tópica.

A revisão do UpToDate (Quadro I), de 2011, aponta para a eficácia dos AINE no alívio da dor associada à inflamação venosa e diminuição significativa da extensão e recorrência da TS em comparação com o placebo. Nenhum AINE mostrou, de forma consistente, ser mais eficaz do que outro no tratamento da TS. Demonstra também haver evidência da maior eficácia da HBPM na redução da extensão do trombo e da recorrência deTS comparativamente ao placebo, não tendo sido encontradas diferenças significativas na eficácia comparativa de doses fixas ou de doses ajustadas ao peso de nadroparina, ou de alta dose versus baixa dose de enoxaparina.

Esta revisão atribui força de recomendação B às seguintes orientações de tratamento:

- Doentes com baixo risco de TVP: AINE como 1. ${ }^{a}$ linha.

- Doentes com alto risco de TVP: Anticoagulação por 4 semanas. HBPM, HNF e AVK mostraram eficácia semelhante.

- Trombo com extensão ao sistema venoso profundo: anticoagulação segundo protocolo de tratamento de TVP. A meta-análise encontrada (Quadro II), da Cochrane e de 2007, revista em 2010, incluiu 24 estudos, com uma amostra total de 2469 casos com TS. Os seus resultados destacam que tanto a HBPM como os AINE reduzem significativamente a progressão da TS e a sua recorrência em cerca de $70 \%$ comparativamente ao placebo. Em comparação com placebo e tratamento tópico, tanto os AINE como a HBPM previnem com maior eficácia eventos tromboembólicos (ETE) e a extensão da TS, e controlam os sintomas locais. A HBPM, quer em doses terapêuticas quer em doses profilácticas, demonstrou igual redução da progressão da TS e prevenção da sua recorrência num período de follow-up de 3 meses. Relativamente à prevenção de ETE, durante o período de tratamento a dose terapêutica mostrou melhor eficácia (77\%) comparativamente à dose profilática (33\%). Porém, esta vantagem esbate-se após a suspensão da terapêutica. Ambas as terapêuticas (HBPM e AINE) parecem apresentar eficácia e segurança semelhantes, destacando-se apenas o facto da terapêutica com AINE triplicar a probabilidade de gastralgia comparativamente ao placebo. É salientado o baixo nível de qualidade metodológica da maioria dos ensaios clínicos, com necessidade de mais ECA para apurar o papel dos AINE e da HBPM, as doses ideais e a duração do tratamento, e até que ponto a terapêutica combinada pode ser mais eficaz que a terapêutica isolada. Os autores sugerem a utilização de HBPM no tratamento da TS, por um período mínimo de um mês (nível de evidência 2).

No ECA seleccionado foram incluídos 50 doentes diagnosticados com TS da veia safena interna, aleatoriamente divididos em 2 grupos de 25 , sendo que o primeiro grupo foi medicado com HBPM e o segundo com HBPM associado a AINE (Quadro III). ${ }^{11}$ Ambos os tratamentos tiveram a duração de 10 dias, tendo sido associado pantoprazol $40 \mathrm{mg}$ id aos dois grupos para protecção gástrica. Dor, hiperémia, sensibilidade local e tamanho do cordão palpável foram medidos e comparados entre os dois grupos. Foi utilizada a escala visual analógica para medição de parâmetros antes e após o tratamento. No segundo grupo houve uma redução estatisticamente significativa da dor e sensibilidade local. Não foram detectadas complicações no primeiro grupo, tendo sido detectado um caso de hematúria no segundo grupo. Os autores deste ECA concluem que os AINE podem ser associados a HBPM para tratamento da TS com melhor alívio da sintomatologia e sugerem 


\begin{tabular}{|c|c|c|c|c|}
\hline Autores & População & Intervenção & Conclusões & $\begin{array}{l}\text { Nível de } \\
\text { evidência }\end{array}$ \\
\hline $\begin{array}{l}\text { Di Nisio M, et al. }{ }^{5} \\
24 \text { ECA }\end{array}$ & $\begin{array}{l}\mathrm{n}=2469 \\
\mathrm{TS}\end{array}$ & $\begin{array}{l}\text { Terapêutica } \\
\text { HBPM, AINE, } \\
\text { tratamento tópico, } \\
\text { tratamento cirúrgico } \\
\text { Evidências } \\
\text { Tanto a HBPM como os } \\
\text { AINE reduzem a } \\
\text { incidência de } \\
\text { recorrências ou } \\
\text { complicações da TS em } \\
\text { aproximadamente } 70 \% \\
\text { em comparação com } \\
\text { placebo e têm eficácia e } \\
\text { segurança similar }\end{array}$ & $\begin{array}{l}\text { Opção } \\
\text { terapêutica } \\
\text { AINE ou } \\
\text { HBPM } \\
\text { Limitações } \\
\text { Necessários } \\
\text { mais estudos } \\
\text { para comparar } \\
\text { estas duas } \\
\text { terapêuticas } \\
\text { Pelos autores } \\
\text { Dose fixa de } \\
\text { HBPM por um } \\
\text { período mínimo } \\
\text { de } 1 \text { mês }\end{array}$ & 2 \\
\hline
\end{tabular}

TS - Tromboflebite superficial

HBPM - Heparina de baixo peso molecular

AINE - Anti-inflamatório não esteróide

que esta terapêutica combinada deve ser considerada no tratamento da TS. Contudo, admitem a necessidade de mais estudos (nível de evidência 2).

\section{CONCLUSÕES}

O limite temporal de três anos utilizado na pesquisa foi definido em relação com a data da última actualização da NOC (2008). O processo de revisão realizado pelos autores da NOC não determinou alterações nas recomendações principais no tratamento da TS. ${ }^{12}$ Este facto revela a escassa investigação nesta área. A integração desta informação foi analisada e justificou a escolha de um limite temporal mais curto.

A revisão realizada permite perceber que a abordagem terapêutica da TS ainda é controversa. A HBPM e os AINE mostraram-se eficazes na melhoria dos sintomas dos doentes com TS em comparação com placebo e tratamento tópico isolado, reduzindo a incidência de recorrências e complicações, e estão recomendados como primeira linha de tratamento (força de recomendação B). Nos casos identificados como tendo fac- tores de risco para complicações, a HBPM em doses intermédias/terapêuticas por um período de pelo menos 4 semanas é opção de primeira linha (força de recomendação B). No entanto, a evidência científica que suporta esta afirmação não é muito robusta, sendo que a maioria dos estudos apresenta baixa qualidade e não são claras qual a dosagem e duração terapêutica mais adequadas, nem qual o possível benefício da combinação de HBPM e AINE. É discutível a opção por um destes fármacos ou mesmo a sua utilização simultânea.

Neste sentido, devem ser realizados ECA com amostras de maiores dimensões e de melhor qualidade metodológica, não apenas utilizando critérios baseados em sinais e sintomas, mas também avaliando o perfil de segurança desta associação em termos de fenómenos hemorrágicos e/ou complicações gástricas, assim como avaliar a recorrência da TS e complicações a longo prazo. Na perspectiva dos autores, a escala visual analógica, utilizada no ECA incluído nesta revisão, é um instrumento pouco indicado para avaliação da hiperémia, sensibilidade local e tamanho do cordão palpável, pois condiciona subjectividade do examinador na avaliação de sinais objectivos. Os autores sugerem que um método de classificação mais objectivo em termos de caracterização será mais indicado. A título de exemplo, o recurso a medição objectiva do tamanho do cordão venoso palpável e da zona de hiperémia poderá ser preconizado.

Em conclusão, são necessários ECA de elevada qualidade, com enfoque na comparação entre AINE e HBPM, para melhor esclarecer as evidências supracitadas e estabelecer forças de recomendação mais consistentes. Adicionalmente, a opção por AINE ou HBPM ou a sua utilização simultânea, assim como a determi- 


\begin{tabular}{|c|c|c|c|c|}
\hline Autores & População & Intervenção & Conclusões & $\begin{array}{l}\text { Nível de } \\
\text { evidência }\end{array}$ \\
\hline Uncu $\mathrm{H}^{11}$ & $\mathrm{n}=50$ & $\begin{array}{l}\text { Terapêutica } \\
25 \text { participantes: }\end{array}$ & $\begin{array}{l}\text { Opção } \\
\text { terapêutica }\end{array}$ & 2 \\
\hline Turquia & $\begin{array}{l}\text { TS da veia } \\
\text { safena interna }\end{array}$ & $\begin{array}{l}\text { Nadroparina } \\
\text { (190 Ul/kg, id) } \\
25 \text { participantes: } \\
\text { Nadroparina } \\
\text { (190 UI/kg, id) } \\
\text { +Acemetacina } \\
\text { (60 mg, 2id) } \\
\text { Evidências } \\
\text { O grupo medicado } \\
\text { com HBPM+AINE } \\
\text { teve melhores } \\
\text { resultados a nível de } \\
\text { alívio sintomático } \\
\text { global, especialmente } \\
\text { na dor e sensibilidade } \\
\text { local }\end{array}$ & $\begin{array}{l}\text { HBPM associado } \\
\text { a AINE } \\
\text { Limitações } \\
\text { Necessários mais } \\
\text { estudos para } \\
\text { comparar estas } \\
\text { duas terapêuticas }\end{array}$ & \\
\hline
\end{tabular}

TS - Tromboflebite superficial HBPM - Heparina de baixo peso molecular AINE - Anti-inflamatório não esteróide
2. Milio $G$, Siragusa $S$, Minà $C$, Amato $C$, Corrado $E$, Grimaudo $S$, et al. Superficial venous thrombosis: prevalence of common genetic risk factors and their role on spreading to deep veins. Thromb Res 2008; 123 (2): 1949.

3. Decousus H, Quéré I, Presles E, Becker F, Barrellier MT, Chanut $M$, et al. Superficial venous thrombosis and venous thromboembolism: a large, prospective epidemiologic study. Ann Intern Med 2010 Feb 16; 152 (4): 218-24.

4. Fernandez L, Scovell S. Superficial thrombophlebitis of the lower extremity. In: Basow DS, editor. UpToDate. Waltham, MA: UpToDate; 2011.

5. Di Nisio M, Wichers IM, Middeldorp S. Treatment for superficial thrombophlebitis of the leg. Cochrane Database of Systematic Reviews 2007, Issue 2. Art. No.: CD004982. DOI: 10.1002/14651858.CD004982.pub3 (version 3 published online in Issue 12, 2010)

6. Hingorani A, Ascher E. Superficial Thrombophlebitis. In: Zelenock GB, Huber TS, Messina $L M$, editors. Mastery of vascular and endovascular surgery. Philadelphia, PA: Lippincott Williams \& Wilkins; 2006. p. 540.

7. Creager MA, Loscalzo J. Vascular diseases of the extremities. In: Kasper DL, Braunwald E, Fauci AS, Hauser SL, Longo DL, Jameson JL, et al., editors. Harrison's Principles of Internal Medicine. 17th ed. New York: McGraw-Hill Medical Publishing Division; 2008

nação das doses e da duração ideais do tratamento, dependerá da realização de ECA que contemplem de modo sistematizado estas variáveis. A melhor caracterização da TS no que diz respeito à sua prevalência em Portugal e o esclarecimento de consequências clínicas relacionadas permitirão reconhecer o grau de importância clínica desta condição. Investigações futuras deverão também equacionar questões relacionadas com o custo-eficácia das opções terapêuticas referidas.

\section{AGRADECIMENTOS}

Agradecemos ao Professor Alberto Hilário Ramos Ferreira Pimenta pela importante colaboração na elaboração deste trabalho, através da revisão de texto.

\section{REFERÊNCIAS BIBLIOGRÁFICAS}

1. Leon L, Giannoukas AD, Dodd D, Chan P, Labropoulos N. Clinical significance of superficial vein thrombosis. Eur J Vasc Endovasc Surg 2005 Jan; 29 (1): 10-7.
8. Ebell MH, Siwek J, Weiss BD, Woolf SH, Susman J, Ewigman B, et al. Strength of recommendation taxonomy (SORT): a patient-centered approach to grading evidence in the medical literature. Am Fam Physician 2004 Feb 1; 69 (3): 548-56.

9. Kearon C, Kahn SR, Agnelli G, Goldhaber S, Raskob GE, Comerota AJ. Antithrombotic therapy for venous thromboembolic disease: American College of Chest Physicians Evidence-Based Clinical Practice Guidelines (8th Edition). Chest 2008 Jun 133 (6 Suppl): 454S-545S.

10. Guyatt $G H$, Oxman AD, Vist GE, Kunz R, Falck-Ytter Y, Alonso-Coello P, et al. GRADE: an emerging consensus on rating quality of evidence and strength of recommendations. BMJ 2008 Apr 26; 336 (7650): 924-6.

11. Uncu H.A comparison of low-molecular-weight heparin and combined therapy of low-molecular-weight heparin with an anti-inflammatory agent in the treatment of superficial vein thrombosis. Phlebology 2009 Apr; 24 (2): 56-60.

12. Büller HR, Agnelli G, Hull RD, Hyers TM, Prins MH, Raskob GE. Antithrombotic therapy for venous thromboembolic disease: the Seventh ACCP Conference on Antithrombotic and Thrombolytic Therapy. Chest 2004 Sep; 126 (3 Suppl): 401S-428S.

13. Braga R, Melo M. Como fazer uma Revisão Baseada na Evidência. Rev Port Clin Geral 2009 Nov-Dez; 25 (6): 660-6. 


\section{CONFLITO DE INTERESSES}

Luiz Miguel Santiago declara que recebeu honorários para a realização de exposições formativas, pelas organizações farmacêuticas Sanofi-Aventis e Solvay-Abbott. Os restantes autores declaram não possuir quaisquer conflitos de interesse.

\author{
ENDEREÇO PARA CORRESPONDÊNCIA \\ Philippe José Couto Botas \\ Rua das Arroteias, N. ${ }^{\circ} 1$ \\ 3105 Pombal \\ E-mail: philippe_botas@hotmail.com
}

Recebido em 12/08/2011

Aceite para publicação em 18/06/2012

\section{ABSTRACT}

\section{MEDICAL TREATMENT OF SUPERFICIAL THROMBOPHLEBITIS OF THE LOWER LIMB: HEPARIN OR ANTI-INFLAMMATORY DRUGS?}

Objectives: The objective of this review is to compare treatment with non-steroidal anti-inflammatory drugs (NSAID) and lowmolecular-weight heparin (LMWH) for superficial thrombophlebitis (ST) of the lower limb.

Data sources: We searched the PubMed database, evidence-based medicine websites, the General Directorate of Health website, the Portuguese Association of General Practitioners website, MGFamiliar.net, and the Index of Portuguese Medical Magazines. Review methods: We selected clinical guidelines (CG), computerized decision support systems (CS), systematic reviews (SR) and original studies, published between January 2008 and May 2011, using the MeSH terms venous thrombosis, heparin, low molecular weight, and anti-inflammatory agents. American Family Physician's Strength of Recommendation Taxonomy (SORT) was used to assess the level of evidence.

Results: 215 articles were obtained. We selected one CG, one CS, one SR and one randomized clinical trial (RCT) for this review. The evidence found suggests that LMWH or NSAIDs are superior to placebo in the treatment of symptoms of ST, and in reducing the incidence of recurrences and complications, without differences in their safety profile in the short term (level of evidence 2). The evidence also confirms anticoagulation as the first line of therapy (level of evidence 2). The simultaneous use of LMWH and NSAID was found to be more effective for symptomatic relief than LMWH alone in one RCT (level of evidence 2). Conclusions: There is evidence for the use of LMWH and NSAID as first-line treatment for ST (SOR B). Anticoagulation for at least 4 weeks is indicated especially when the following criteria are present: anatomical proximity of the thrombus to the deep venous system and medical risk factors for deep vein thrombosis (SOR B). More randomized clinical trials are needed, comparing LMWH and NSAID, evaluating the simultaneous use of these drugs, and assessing the proper dose and duration of treatment.

Keywords: thrombophlebitis, heparin, anti-inflammatory, therapeutics. 